\title{
RQWDFB: ANALYSIS OF RETRIAL QUEUES WITH DELAYED FEEDBACK
}

\author{
AGASSI MELIKOV, SEVINJ ALIYEVA, AND JÁNOS SZTRIK
}

Received 13 May, 2020

\begin{abstract}
The models of retrial queues $\mathrm{M} / \mathrm{M} / \mathrm{K} / \mathrm{K}$ with delayed feedback are considered. If upon arrival of primary call ( $p$-call) there are free channels then it occupies one of them for a some exponentially distributed time; otherwise, it in accordance to Bernoulli scheme either leaves the system forever or go to orbit for retrial calls ( $r$-calls). After the customer is served completely, it will decide based on Bernoulli scheme either to join the orbit for re-service after some delay (delayed feedback) or to leave the system forever. If upon arrival $r$-call there is a free server, then the arriving call begins to be served immediately; otherwise, it in accordance to Bernoulli scheme either leaves the orbit or return to orbit. An approximate method to calculation of the steady-state probabilities of the appropriate two-dimensional Markov chain as well as performance measures of the system is developed. Results of numerical experiments are demonstrated.
\end{abstract}

2010 Mathematics Subject Classification: 60J10; 60K25; 90B05; $90 \mathrm{~B} 22$

Keywords: multi-channel queuing system, retrial calls, delayed feedback, finite and infinite orbits, balking from orbit, calculation method

\section{INTRODUCTION}

Retrial of calls are a common phenomenon in queuing systems. In retrial queuing (RQ) systems arriving new calls who find all servers and waiting room occupied is decide to leave the system forever or to join the retrial group to try again for their service after a random period of time. Performance modeling of RQ systems has attracted many researchers since they adequately describe many real life problems, e.g. in switching systems, telecommunication networks, computer networks, inventory systems etc. $[1,10]$. For comprehensive survey and list of references in this field see $[11,23]$.

Retrial phenomenon is observed in queuing systems with feedback (FB) as well. In such systems serviced calls require repeated service depending on some factors, e.g. quality of service, response time in the system, etc. For the state of the art of the theoretical and practical researches in queuing systems with FB see [19].

The work of János Sztrik is supported by the EFOP-3.6.1-16-2016-00022 project. The project is co-financed by the European Union and the European Social Fund. 
Note that almost all papers on RQ have considered the systems without feedback. However, retrial queuing systems with delayed feedback (RQwDFB) are suitable models for many practical situations, e.g. multiple access telecommunication systems, where data that is transmitted erroneously are sent again can be modeled as retrial queue with feedback.

In the literature there are few works devoted to study RQwDFB. It seems that $\mathrm{RQwDFB}$ for the first time has been investigated in paper [5]. In this paper authors investigated an RQwDFB $M / M / c / c$ with infinite orbit capacity when $c=1,2$. An arriving call is served immediately if one of the servers is idle, and if all servers are busy, it will either join the retrial group to try again after a random period of time or leave the system forever without service. On retrials, each customer is treated the same as a primary customer. If retrial call finds free servers, it receives service immediately, and if all the servers are busy, it will either leave the system forever or join the retrial group. All calls in the retrial group act independently of each other. The retrial time is exponentially distributed with finite mean and is independent of all previous retrial times and all other stochastic processes in the system. After the call is served completely, it will decide either to join the retrial group again for another service or to leave the system forever. They found the joint probability generating function (PGF) of the number of busy servers and the number of customers in the retrial group for $c=1,2$.

In [21] considered an RQwDFB $M / G / 1 / 1$ with single server vacation and constant retrial rate where the server is subjected to starting failures. It is assumed that the retrial time is governed by an arbitrary distribution. The server leaves for a vacation as soon as the system becomes empty. When the server returns from the vacation and finds no calls, he waits free for the first customer to arrive from outside the system. If the server is started successfully (with a certain probability), the call gets service immediately; otherwise, the repair for the server starts immediately and the call must leave for the orbit and make a retrial later. The system size distribution at random points and various performance measures are derived.

In [2,3,8] considered Markovian models of single server RQwDFB. Models with two types of calls under non-pre-emptive and pre-emptive priority service disciplines are investigated in [3] and [2], respectively. In both papers [3] and [2] the retrial, loss and feedback allowed for low priority calls only. If the server is free at the time of the arrival of low priority call, then an incoming call immediately begins to be serviced; after completion of service, low priority call either may re-join the orbit of infinite size or leaves the system. If the server is busy then the arriving low priority call goes to orbit and becomes a source of repeated calls. If the server is free at the time of the arrival of high priority customer, then the arriving call begins to be served immediately and high priority customer leaves the system after service completion. In [3] the non-pre-emptive priority service discipline is used, i.e. if the server is engaging with low priority call and the higher priority call comes then the 
high priority call will get service only after completion of the service of low priority call who is in service. In [2] the pre-emptive priority service is used, i.e. if the server is engaging with low priority call and the higher priority call enters then the high priority call will get service immediately and the low priority call who is in service goes to orbit without completion of his service. The model of single server RQwDFB with batch service under multiple server vacations is considered in [8]. It is assumed that upon completion the service batch of calls in accordance to Bernoulli scheme either may join the queue or leaves the system. If after completion of the service number of calls in the queue less than a given threshold, then server leaves for a multiple vacation period with exponential distribution. The arriving call in accordance to Bernoulli scheme either may join the queue or leaves the system when server is busy or in vacation.

Model of RQwDFB $M / M / 1 / N, N<\propto$, with two orbits is considered in [4]. If upon arrival, the calls find the queue full, then they decide to stay for an attempt to get service or leave the system. Arriving customers who decide to stay for an attempt have to choose one of the orbits in accordance with the probabilities that depend on thresholds in orbits. After the call is served completely, it may decide to either attempt for re-service or leave the system forever. In this case, the selection of one of the orbits occurs according to the Bernoulli scheme.

Recently in [25] considered an RQwDFB $M / G / 1$ with negative calls under working vacation and working breakdowns. The PGFs for the numbers of calls in the orbit under different server status are derived. Various system performance measures and some special cases were discussed.

To study models of RQwDFB in [4, 5, 8, 21, 25] PGF approach is used while in $[2,3]$ matrix-geometric method [22] is applied.

Note that indicated above works devoted to single-server RQwDFBs. Moreover, in these works it is assumed that the retrial and feedback probabilities are constant quantities, i.e. they do not depend on the state of the system. These assumptions significantly limit the application areas of the proposed models, since in practice RQwDFB contains many servers and in these systems decisions about going into orbit or leaving orbit without getting re-service often depend on the current state of the system.

Models of multi-server RQwDFBs, in contrast its single-server counterpart, are not studied (exception is [5] in which two-server system is investigated as well). This paper is motivated by the work [5]. The novelty of our contribution is threefold. Firstly, we consider models of multi-server RQwDFB with both finite and infinite capacity of orbit. Secondly, we take into account that retrial, feedback probabilities as well as balking probabilities from the orbit are state-dependent; in other words, we assume that both arrived and departure calls as well as retrial calls have information about number of calls in orbit, i.e. appropriate decisions depend on number of calls in orbit. Thirdly, we propose an approximate method to study the proposed models 
of RQwDFB. The developed method allows calculate the steady-state probabilities as well as performance measures of the models under study for arbitrary number of channels and capacity of orbits for retrial and feedback calls. Moreover, calculations are realized by explicit formulas that contains tabulated quantities.

Proposed here approach to study models of RQwDFB based on the idea of theory of space merging of Markov chains [12]. The space-merging algorithm (SMA) for the first time was used to calculation of the steady-state probabilities of the twodimensional Markov chain (2D MC) in $[6,17,18]$. Recently hierarchical SMA to study 3D MC is developed in [13]. In last decade many researchers develop similar SMAs to study 2D MC in various areas of applications [7,9,14-16,24]. In all papers, authors note high efficiency of SMA (in both sense of accuracy and complexity) in comparison with other numerical methods.

This paper is structured as follows. Basic mathematical model of the investigated RQwDFB is described in Section 2. Generator matrix of the constructed 2D MC is created in Section 3. Here exact method to calculate its steady-state probabilities is indicated and explicit formulas for performance measures of the investigated system is developed as well. In Section 4, approximate method for solving indicated above problems is proposed. Results of numerical experiments that show high accuracy of the approximate method as well as behavior of performance measures versus system parameters are demonstrated in Section 5. Conclusions are given in Section 6.

\section{MODEL DESCRIPTION}

First consider the model with finite capacity of orbit, i.e. let maximum capacity of orbit is $R, R<\infty$. The system contains $K>1$ identical channels and a new arriving calls (or primary calls, $p$-calls) form Poisson flow with rate $\lambda$. An incoming $p$-call is accepted for service if at this moment there is a free channel; otherwise, i.e. if upon arrival of $p$-call all channels are busy, then it either go to orbit with a probability (w.p.) $\alpha_{r}$ or leave the system w.p. $1-\alpha_{r}$ when there are $r$ calls in the orbit, $\alpha_{R}=0$.

Calls from orbit ( $r$-calls) independently each other generates request to service. It is assumed that times between arrivals of $r$-calls have common exponential distribution with mean $\eta^{-1}$, i.e. if there are $r$ calls in orbit then arrival intensity is $r \eta$ (linear retrial rate). If upon arrival of $r$-call all channels are busy, then it either leave the orbit w.p. $\sigma_{r}$ or return to orbit w.p. $1-\sigma_{r}$ when there are $r$ calls in the orbit.

After completion of the service process, the call, according to the Bernoulli scheme, either w.p. $\beta_{r}$ go to orbit in order to requires repeated service after some positive delay, or with a complementary probability $1-\beta_{r}$ leaves the system forever when there are $r$ calls in the orbit, $\beta_{R}=0$.

Service times for both types of $p$-calls and $r$-calls are independent and identically distributed (i.i.d.) random variables (r.v.) and suppose that their distribution function is exponential one with a common average $1 / \mu$. 
It is assumed that the arriving of $p$-calls from outside, the arriving of $r$-calls from orbit and service of calls, are independent of each other.

The problem is to determine the steady-state probabilities of a given system. The solution of this problem allows us to find the following performance measures of the system: loss probabilities of $x$-calls, $P_{x}, x \in\{p, r\}$; the average number of busy channels $\left(K_{a v}\right)$ and the average number of $r$-calls in orbit $\left(L_{r}\right)$.

\section{GENERATION OF Q-MATRIX AND FORMULAS FOR PERFORMANCE MEASURES}

First consider model with finite capacity of orbit. The state of the system at arbitrary moment of the time is defined by two-dimensional vector $(b, r)$, where $b$ is total number of busy channels and $r$ is number of $r$-calls in orbit. Taking into account the type of distribution functions of all r.v. that are involved in the formation of the model we conclude that the state space of the appropriate two-dimensional Markov chain (2D MC) is determined as follows:

$$
S=\{1,2, \ldots, K\} \times\{0,1, \ldots, R\} .
$$

Transition intensity from state $(b, r)$ to state $\left(b^{\prime}, r^{\prime}\right)$ is denoted by $q\left((b, r),\left(b^{\prime}, r^{\prime}\right)\right)$. These quantities are determined as follows:

For case $0 \leq b \leq K-1$ :

$$
q\left((b, r),\left(b^{\prime}, r^{\prime}\right)\right)= \begin{cases}\lambda, & \text { if }\left(b^{\prime}, r^{\prime}\right)=(b+1, r), \\ b \mu \beta_{r}, & \text { if } r<R,\left(b^{\prime}, r^{\prime}\right)=(b-1, r+1), \\ b \mu\left(1-\beta_{r}\right), & \text { if } r<R,\left(b^{\prime}, r^{\prime}\right)=(b-1, r), \\ b \mu, & \text { if } r=R,\left(b^{\prime}, r^{\prime}\right)=(b-1, R), \\ r \eta, & \text { if }\left(b^{\prime}, r^{\prime}\right)=(b+1, r-1) .\end{cases}
$$

For case $b=K$ :

$$
q\left((K, r),\left(b^{\prime}, r^{\prime}\right)\right)= \begin{cases}\lambda \alpha_{r}, & \text { if } r<R,\left(b^{\prime}, r^{\prime}\right)=(K, r+1), \\ K \mu \beta_{r}, & \text { if } r<R,\left(b^{\prime}, r^{\prime}\right)=(K-1, r+1), \\ K \mu\left(1-\beta_{r}\right), & \text { if } r<R,\left(b^{\prime}, r^{\prime}=(K-1, r),\right. \\ K \mu, & \text { if } r=R,\left(b^{\prime}, r^{\prime}\right)=(K-1, R), \\ r \eta \sigma_{r}, & \text { if }\left(b^{\prime}, r^{\prime}\right)=(b+1, r-1) .\end{cases}
$$

From relations (3.2) and (3.3) we conclude that all states of space (3.1) are communicated each other, i.e. steady-state probabilities are existing. Let $p(b, r)$ denote stationary probability of state $(b, r) \in S$.

The performance measures of the system are determined as follows:

$$
\begin{aligned}
K_{a v} & =\sum_{b=1}^{K} b \sum_{r=0}^{R} p(b, r) ; \\
L_{r} & =\sum_{r=1}^{R} r \sum_{b=0}^{K} p(b, r) ;
\end{aligned}
$$




$$
\begin{aligned}
P_{p} & =p(K, R)+\sum_{r=0}^{R-1}\left(1-\alpha_{r}\right) p(K, r) ; \\
P_{r} & =\sum_{r=1}^{R} \sigma_{r} p(K, r) .
\end{aligned}
$$

For the model with moderate dimension of state space (3.1) the steady-state probabilities might be calculated from system of equilibrium equations (SEE). Due to the complex structure of the obtained GM (see formulas (3.2) and (3.3)), it is not possible to find analytical solution of SEE. To solve the indicated problem Neuts' matrix-geometric method and its modifications as well as spectral expansion method [20] might be used. However, these methods often suffer from computational instability for large values of number of channels and orbit capacity. Therefore, an alternative (approximate) method for solving this problem is given below. It is based on the space merging approach for multidimensional MCs and it allows calculate the steady-state probabilities and performance metrics via explicit formulas. Moreover, the obtained formulas contain quantities that are tabulated even, i.e. Erlang's loss formulas etc.

\section{Approximate Method Based on Space Merging Approach}

For the correct application of the developed method, it is required satisfying the following condition: the state space (3.1) is decomposed into classes in a such a way that the transitions between states within classes are much larger than transitions between states of different classes. This condition is fulfilled under certain ratios of the initial parameters of the system. Let us assume that arrival rate of $p$-calls is much larger than arrival rate of $r$-calls, i.e. below we assume that the following conditions are fulfilled: $\eta<<\lambda$.

When fulfilling this assumption, we consider the following splitting of the state space (3.1):

$$
S=\bigcup_{r=0}^{R} S_{r}, \quad \quad S_{r} \bigcap S_{r^{\prime}}=\varnothing, \quad r \neq r^{\prime},
$$

where $S_{r}=\{(b, r) \in S: b=0,1, \ldots, K\}, r=0,1, \ldots, R$.

Under accepted above assumptions the transitions between states within classes $S_{r}$ are much larger than transitions classes between states of different classes. Based on splitting (4.1) in the state space (3.1), the following merge function is defined:

$$
U(b, r)=<r>, \quad(b, r) \in S_{r},
$$

where $\langle r\rangle$ is the merged state which includes all states from the class $S_{r}$, $r=0,1, \ldots, R$. We denote $\Omega=\{\langle r\rangle: r=0,1, \ldots, R\}$. 
Approximate values of the steady-state probabilities of the initial 2D MC are denoted by $\tilde{p}(b, r)$. They are determined as follows:

$$
\tilde{p}(b, r)=\rho_{r}(b) \pi(<r>),
$$

where $\rho_{r}(b)$ is the probability of state $(b, r)$ within splitting model with state space $S_{r}, \pi(<r>)$ is the probability of merged state $<r>\in \Omega$.

Remark 1 . Because of in splitting model with state space $S_{r}$ states $(b, r)$ are uniquely defined by component $b$, hereinafter these states are denoted as scalar $b$, $b=0,1, \ldots, K$.

From (3.2) and (3.3) we conclude that the probabilities of the states of splitting models with space $S_{r}, 0 \leq r \leq R-1$, coincide with the state probabilities of the onedimensional birth-death process (1D BDP) in which the birth intensity is equal to $\lambda$ and the death intensity in the state $b$ is equal to $b \mu\left(1-\beta_{r}\right)$. So, steady-state probabilities $\rho_{r}(b), 0 \leq r \leq R-1$, coincide with steady-state probabilities of Erlang's model $M / M / K / K$ with load $\mathrm{v}_{r}=\lambda / \mu\left(1-\beta_{r}\right)$, i.e.

$$
\rho_{r}(b)=\frac{v_{r}^{b}}{b !} / \sum_{i=0}^{K} \frac{v_{r}^{i}}{i !}, b=0,1, \ldots, K .
$$

By same way we conclude that the probabilities of the states of splitting model with space $S_{R}$ coincide with the state probabilities of the 1D BDP in which the birth intensity is equal to $\lambda$ and the death intensity in the state $b$ is equal to $b \mu$. In other words, steady-state probabilities $\rho_{R}(b), 0 \leq b \leq K$, coincide with steady-state probabilities of Erlang's model $M / M / K / K$ with load $\nu=\lambda / \mu$.

Merged model with state space $\Omega$ represents $1 \mathrm{D}$ BDP as well. Transition rates between its states denote by $\left.q\left(<r>,<r^{\prime}\right\rangle\right)$. It is clear that $\left.q\left(<r>,<r^{\prime}\right\rangle\right)=$ 0 if $\left|r-r^{\prime}\right|>1$. Positive transition rates are calculated as follows:

For $0 \leq r \leq R-1$ :

$$
\begin{aligned}
q(<r>,<r+1>) & =\lambda \alpha_{r} \rho_{r}(K)+\mu \beta_{r} \sum_{b=1}^{K} b \rho_{r}(b) \\
& =\lambda \alpha_{r} E_{B}\left(v_{r}, K\right)+\mu \beta_{r} B_{a v}\left(v_{r}, K\right) .
\end{aligned}
$$

For $1 \leq r \leq R$ :

$$
\begin{aligned}
q(<r>,<r-1>) & =r \eta \sum_{b=1}^{K-1} \rho_{r}(b)+r \eta \sigma_{r} \rho_{r}(K) \\
& =r \eta\left(1-\left(1-\sigma_{r}\right) E_{B}\left(v_{r}, K\right)\right) .
\end{aligned}
$$

Hereinafter, $E_{B}(x, K)$ and $B_{a v}(x, K)$ denote the Erlang B-formula and the average number of busy channels for the $M / M / K / K$ system with a load $x$, respectively, i.e.

$$
E_{B}(x, K)=\frac{x^{K}}{K !} / \sum_{k=0}^{K} \frac{x^{k}}{k !} ; B_{a v}(x, K)=x\left(1-E_{B}(x, K)\right) .
$$


Therefore, from relations (4.5) and (4.6) we conclude that the probabilities of merged states are calculated by classical formulae for 1D BDP:

$$
\pi(<r>)=\prod_{i=0}^{r-1} \frac{\Lambda_{i}}{\mathrm{M}_{i+1}} \pi(<0>), r=1, \ldots, R,
$$

where $\Lambda_{i}=q(<i>,<i+1>), \mathrm{M}_{i}=q(<i>,<i-1>)$ and $\pi(<0>)$ is found from normalizing condition, i.e. $\sum_{r=0}^{R} \pi(<r>)=1$.

Finally, taking into account relations (4.3), (4.4) and (4.7), approximate values of the steady-state of the initial 2D MC are found. Further, after certain mathematical transformations, we obtain the following approximate formulas for calculating the performance measures (3.4)-(3.7) of the system under study:

$$
\begin{aligned}
K_{a v} & \approx \sum_{b=1}^{K} b \sum_{r=0}^{R} \rho_{r}(b) \pi(<r>) ; \\
L_{r} & \approx \sum_{r=1}^{R} r \pi(<r>) ; \\
P_{p} & \approx E_{B}\left(v_{R}, K\right) \pi(<R>)+\sum_{r=0}^{R-1}\left(1-\alpha_{r}\right) E_{B}\left(v_{r}, K\right) \pi(<r>) ; \\
P_{r} & \approx \sum_{r=1}^{R} \sigma_{r} E_{B}\left(v_{r}, K\right) \pi(<r>) .
\end{aligned}
$$

Case of infinite orbit capacity. Note that in the case of constants probabilities $\alpha_{r}$, $\beta_{r}$ and $\sigma_{r}$ for all the possible $r$, the formulas (4.8)-(4.11) are even more simplified. Moreover, in this case it is possible to obtain explicit solutions for models with an infinite orbit capacity.

So, for a model with an infinite orbit capacity $(R=\infty)$, the state probabilities inside all splitting models with space $S_{r}, r=0,1,2, \ldots$ coincide with the state probabilities of the Erlang model $M / M / K / K$ with load $v /(1-\beta)$ (see formula (4.4)). From relations (4.5) and (4.6) we conclude that for this model the probabilities of merged states coincide with the probabilities of the states of the model $M / M / \infty$ with the load $\theta=\Lambda / \Psi$, i.e.

$$
\pi(<r>)=\frac{\theta^{r}}{r !} \exp (-\theta), \quad r=0,1,2, \ldots
$$

where $\Lambda=\lambda \alpha E_{B}\left(\frac{v}{1-\beta}, K\right)+\mu \beta B_{a v}\left(\frac{v}{1-\beta}, K\right), \mathrm{M}=\eta\left(1-(1-\sigma) E_{B}\left(\frac{v}{1-\beta}, K\right)\right)$.

Then by using (4.12) from formulas (4.8)-(4.11) we obtain that the performance measures of the model with an infinite orbit capacity are approximately determined as follows:

$$
K_{a v} \approx B_{a v}\left(\frac{v}{1-\beta}, K\right)
$$




$$
\begin{aligned}
& L_{r} \approx \sum_{r=1}^{R} r \pi(<r>)=\theta \\
& P_{p} \approx(1-\alpha) E_{B}\left(\frac{v}{1-\beta}, K\right) \\
& P_{r} \approx \sigma(1-\exp (-\theta)) E_{B}\left(\frac{v}{1-\beta}, K\right) .
\end{aligned}
$$

\section{NUMERICAL RESUlts}

Here results of numerical experiments are demonstrated. The goal of these experiments is investigation of the accuracy of the developed approximate formulas. For concreteness, we investigate the behavior of the accuracy versus the rate of $r$-calls $(\eta)$.

As a measure of proximity of two distributions of steady-state probabilities (exact and approximate) the following norms are used:

Cosine similarity:

$$
\|\mathrm{N}\|_{1}=\frac{\sum_{(b, r) \in S} p(b, r) \tilde{p}(b, r)}{\left(\sum_{(b, r) \in S}(p(b, r))^{2}\right)^{\frac{1}{2}}\left(\sum_{(b, r) \in S}(\tilde{p}(b, r))^{2}\right)^{\frac{1}{2}}} ;
$$

Euclidean distance:

$$
\|\mathrm{N}\|_{2}=\left(\sum_{(b, r) \in S}(p(b, r)-\tilde{p}(b, r))^{2}\right)^{\frac{1}{2}} .
$$

Remark 2. In general, cosine similarity norm (5.1) is used to estimate the orientation of two vectors. However, in our experiments it estimates not only the orientation of the steady-state probability vectors in various (exact and approximate) approaches, as well as their Euclidean proximity. This is because, according to the normalization condition, the ends of these two vectors are in the same hyper plane of the Euclidean space of dimension $(K+1)(R+1)$, i.e. $\sum_{(b, r) \in S} p(b, r)=\sum_{(b, r) \in S} \tilde{p}(b, r)=1$. Nonetheless, here values of Euclidean distance is presented as well.

Firstly, consider results of numerical experiments for the model with finite capacity of orbit. In these experiments it is assumed that retrial and feedback probabilities as well as balking probabilities from the orbit are state-dependent and these parameters satisfies the following conditions: $0<\alpha_{r+1} \leq \alpha_{r}<1 ; 0<\beta_{r+1} \leq \beta_{r}<1$; $0<\sigma_{r} \leq \sigma_{r+1}<1$. For concreteness, we assume that $\alpha_{r}=1 /(r+1)$; $\beta_{r}=1 /\left(r^{2}+1\right) ; \sigma_{r}=r /(r+1)$.

Below in all experiments (for both models with finite and infinite capacity of orbit) we assume that $K=3, \lambda=40$. And in experiments for model with finite capacity of orbit it is assumed that $R=20$. 
In Figure 1 the dependence of similarity norms (5.1) and (5.2) on the rate of $r$ calls is demonstrated. Exact values of the steady-state probabilities are calculated from the corresponding SEE. From these graphs we conclude that the steady-state probabilities are calculated with high accuracy, i.e. in wide range of changing rate of $r$-calls the value of similarity norm (5.1) is close to one (see Figure 1A) while Euclidean distance (5.2) is small enough (see Figure 1B).

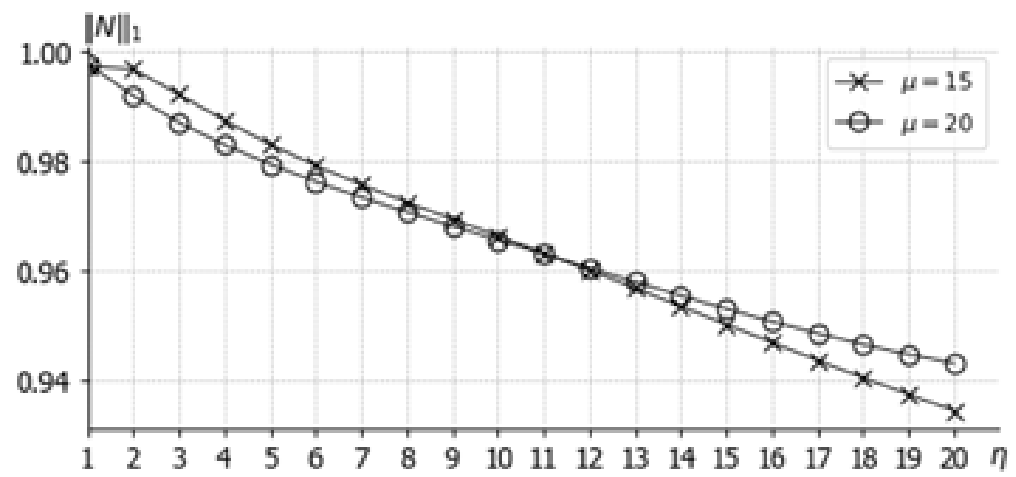

(A)

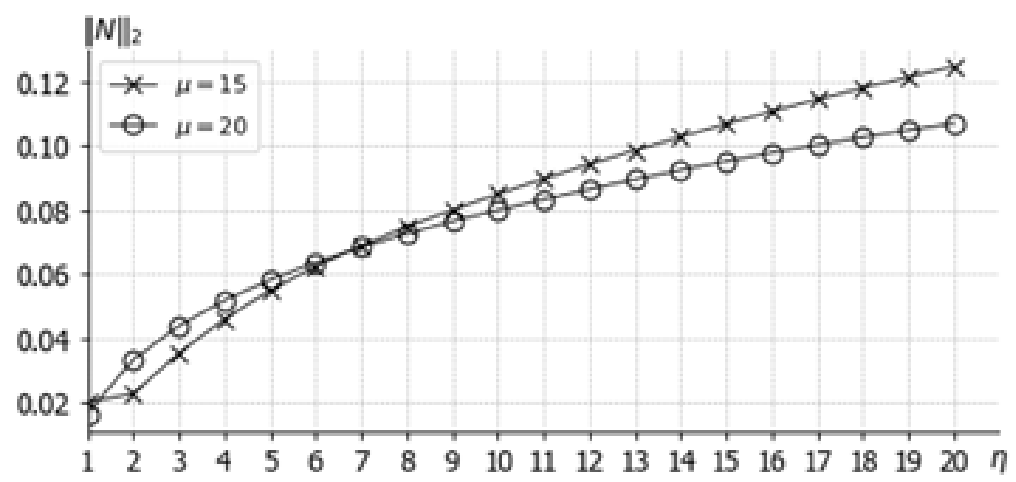

(B)

FIGURE 1. Dependence norms (5.1) (A) and (5.2) (B) on parameter $\eta$ for the model with finite capacity of orbit

It is important to note that when the rate of $r$-calls increases accuracy of calculation of the steady-state probabilities decreases, i.e. the norm (5.1) is decreasing function versus to the rate of $r$-calls while norm (5.2) increases relative to the indicated parameter. These facts were expected since when the rate of $r$-calls is small then the transitions between states of different classes $S_{r}, r=0,1, \ldots, R$, in splitting (4.1) 
is rare, i.e. accuracy of proposed formulas becomes very high. Note that values of norms (5.1) and (5.2) are almost independent on service rate.

Now consider the results of numerical experiments for the model with infinite capacity of orbit, i.e. when $R=\infty$. In these experiments we assume that $\alpha=0.3$, $\beta=0.1, \sigma=0.4$.

The corresponding results for the dependence of similarity norms (5.1) and (5.2) on the rate of $r$-calls are shown in Figure 2.

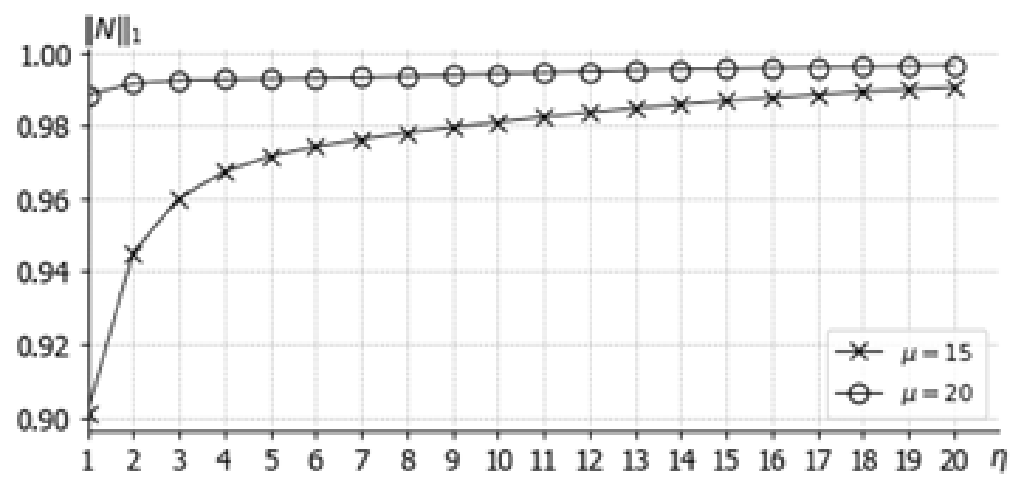

(A)

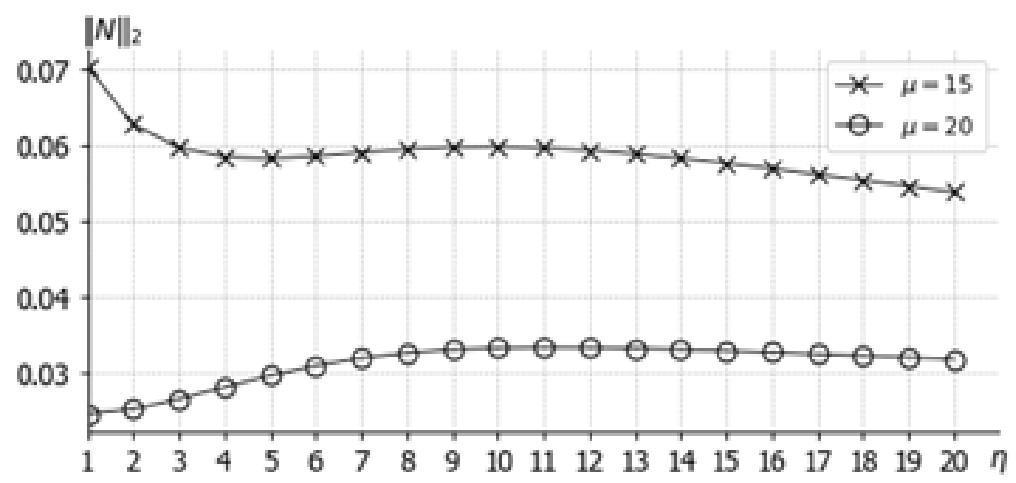

(B)

FIGURE 2. Dependence norms (5.1) (A) and (5.2) (B) on parameter $\eta$ for the model with infinite capacity of orbit

It can be seen from the graphs that in this model accuracy of calculation of approximate values of steady-state probabilities is much better than in model with finite capacity of orbit (see Figure 1). Here exact values of the steady-state probabilities are calculated from the corresponding SEE by using the Python SciPy software package. 
We choose $R$ to be so large that state probabilities $p(b, R), b=\overline{0,3}$, are equal to the machine zero (in our experiments $R=100$ ). In this model values of norms (5.1) and (5.2) are essentially dependent on service rate, i.e. the higher the service intensity, the high value of norm (5.1) and lower value of norm (5.2).

\section{CONCLUSiON}

The paper proposes mathematical models of RQwDFB in which the probabilities of retrial, feedback and balking from the orbit depend on the current number of calls in the orbit. We studied both models with common finite and infinite orbit capacity for retrial and feedback calls and developed exact and approximate methods for calculating their performance measures.

As directions for further research, it is proposed to study similar models in which retrial and feedback calls are distinguished. Of particular interest are also the problems of optimizing RQwDFB regarding the selected criteria for the quality of their functioning. An important area of further research is also the study of the possibility of using special functions [26] to solve the problems investigated here. These problems are the subject of special studies.

\section{ACKNOWLEDGMENT}

The authors are very grateful to the anonymous reviewer for valuable comments and suggestions which improved the quality and presentation of the paper.

Conflicts of interest/Competing interests: The authors declare that they have no conflict of interest.

\section{REFERENCES}

[1] J. R. Artalejo and A. Gómez-Corral, "Retrial queueing systems: A computational approach," Springer-London, 2008, doi: 10.1007/978-3-540-78725-9.

[2] G. Ayyapan, A. Subramanian, A M G, and G. Sekar, "M/M/1 retrial queuing system with loss and feedback under pre-emptive priority service," International Journal of Computer Applications, vol. 2, pp. 27-34, 2010, doi: 10.5120/672-945.

[3] G. Ayyapan, A. M. G. Subramanian, and G. Sekar, "M/M/1 retrial queueing system with loss and feedback under non-pre-emptive priority service by matrix geometric method," Applied Mathematical Sciences, vol. 4, pp. 2379-2389, 2010.

[4] A. Bouchentouf and F. Belarbi, "Performance evaluation of two markovian retrial queueing model with balking and feedback," Acta Universitatis Sapientiae, Mathematica, vol. 5, pp. 132-146, 2013, doi: 10.2478/ ausm-2014-0009.

[5] B. Choi, Y. Kim, and Y. Lee, "The M/M/c retrial queue with geometric loss and feedback," Computers \& Mathematics with Applications, vol. 36, pp. 41-52, 1998, doi: 10.1016/ S0898-1221(98) 00160-6.

[6] B. D. Choi, A. Z. Melikov, and A. M. Velibekov, "A simple numerical approximation of joint probabilities of calls in service and calls in the retrial group in a picocell," Applied and Computational Mathematics, vol. 7, pp. 21-28, 2008.

[7] B. P. Crawford, "Approximate analysis of an unreliable $M / M / 2$ retrial queue," Air Force Institute of Technology, Ohio, Tech. Rep., 2007. 
[8] G. Devipriya, G. Ayyappan, and A. M. G. Subramanian, "Analysis of single server queueing system with batch service under multiple vacations with loss and feedback," Mathematical Theory and Modeling, vol. 4, pp. 79-90, 2014.

[9] M. Elhaddad and F. Belarbi, "On the analysis of unreliable markovian multiserver queue with retrials and impatience," Mathematical Sciences and Applications E-Notes, vol. 7, pp. 205-217, 2019, doi: 10.36753/ mathenot.634506.

[10] G. Falin and J. G. C. Templeton, Retrial queues. London: Chapman \& Hall, $1997 . \quad$ doi: 10.1007/BF02564732.

[11] J. Kim and B. Kim, "A survey of retrial queueing systems," Annals of Operations Research, vol. 247, pp. 3-36, 2016, doi: 10.1007/s10479-015-2038-7.

[12] V. S. Korolyuk and V. V. Korolyuk, Stochastic models of systems. Boston: Kluwer Academic Publishers, 1999.

[13] V. S. Korolyuk, A. Z. Melikov, L. A. Ponomarenko, and A. M. Rustamov, "Asymptotic analysis of the system with server vacation and perishable inventory," Cybernetics and Systems Analysis, vol. 53, pp. 543-553, 2017, doi: 10.1007/s10559-017-9956-0.

[14] C. C. Liang and H. Luh, "Cost estimation queuing model for large-scale file delivery service," International Journal of Electronic Commerce Studies, vol. 2, pp. 19-34, 2011.

[15] C. C. Liang and H. Luh, "Optimal services for content delivery based on business priority," Journal of the Chinese Institute of Engineers, vol. 36, pp. 422-440, 2013.

[16] C. C. Liang and H. Luh, "Solving two-dimensional markov chain model for call centers," Industrial Management \& Data Systems, vol. 115, pp. 901-922, 2015, doi: 10.1108/IMDS-12-20140363.

[17] A. Z. Melikov and A. T. Babayev, "Refined approximations for performance analysis and optimization of queueing model with guard channels for handovers in cellular networks," Computer Communications, vol. 29, pp. 1386-1392, 2006, doi: 10.1016/j.comcom.2005.08.013.

[18] A. Z. Melikov and M. I. Fattakhova, "Computational algorithms to optimization of buffer allocation strategies in a packet switching networks," Applied and Computational Mathematics, vol. 1, pp. 51-58, 2002.

[19] A. Z. Melikov, J. Sztrik, and S. H. Aliyeva, "Analysis of queueing system MMPP/M/K/K with delayed feedback," Mathematics, vol. 7, p. 1128, 2019, doi: 10.3390/math/7111128.

[20] I. Mitrani and R. Chakka, "Spectral expansion solution for a class of Markov models: Application and comparison with the matrix-geometric method," Performance Evaluation, vol. 23, pp. 241260, 1995, doi: 10.1016/0166-5316(94)00025-F.

[21] G. S. Mokaddis, S. A. Metwally, and B. M. Zaki, "A feedback retrial queuing system with starting failures and single vacation," Tamkang Journal of Science and Engineering, vol. 10, pp. 183-192, 2007.

[22] M. F. Neuts, "Matrix-geometric solutions in stochastic models: an algorithmic approach," Johns Hopkins University, Baltimore, 1981.

[23] T. Phung-Duc, "Retrial queueing models: A survey on theory and applications," arXiv:1906.09560, vol. 1, 2019.

[24] L. Raiah and N. Oukid, "An M/M/2 retrial queue with breakdowns and repairs," Romanian Journal of Mathematics and Computer Science, vol. 17, pp. 11-20, 2017.

[25] P. Rajadurai, M. Sundararaman, and D. Narasimhan, "Performance analysis of an $M / G / 1$ retrial G-queue with feedback under working breakdown services." Songklanakarin Journal of Science \& Technology, vol. 42, pp. 236-247, 2020.

[26] H. M. Srivastava and B. R. K. Kashyap, "Special functions in queuing theory and related stochastic processes.” New York: Academic Press, 1982. 
Authors' addresses

\section{Agassi Melikov}

Institute of Control Systems, National Academy of Science, Baku AZ1141, Azerbaijan

E-mail address: agassi.melikov@gmail.com

\section{Sevinj Aliyeva}

Information Technology and Programming, Applied Mathematics and Cybernetics, Baku State University, Baku AZ1141, Azerbaijan

E-mail address: sealiyeva.info

\section{János Sztrik}

(Corresponding author) Department of Informatics and Networks, Faculty of Informatics, University of Debrecen, Debrecen 4032, Hungary

E-mail address: sztrik. janos@inf. unideb.hu 